\title{
Three new CYP450 from Betula platyphylla Suk. with 28 oxidation function catalyze the conversion of lupineol to betulinic acid
}

jie yang ${ }^{1}$, ying li $^{1}$, yuqi zhang ${ }^{1}$, lu sun $^{1}$, siyao wang $^{1}$, jialei xiao ${ }^{2}$, yaguang zhan ${ }^{1}$, and jing Yin $^{1}$

${ }^{1}$ Northeast Forestry University

${ }^{2}$ Northeast Agricultural University

April 28, 2020

\begin{abstract}
Birch(Betula platyphylla Suk.) bark contains important pentacyclic triterpenes as betulin and betulinic acid, which play important functions in anti-tumor and anti-HIV.Cytochrome P450 monooxygenase(CYP450) is essential for the diversification and functional modification of the triterpene skeleton.In this study, five new $C Y P 450$ genes were cloned from birch with ORF lengths of $1284 \mathrm{bp}, 1533 \mathrm{bp}, 1188 \mathrm{bp}, 1704 \mathrm{bp}$, and $1539 \mathrm{bp}$, respectively.Phylogenetic tree analysis shown that five $B p C Y P 450$ genes are located in five subfamilies, named CYP94B89, CYP89S1, CYP97B62, CYP86B54, and CYP86A182.The expression characteristics of five $C Y P 450$ genes in different tissues and their responses to different stresses(MeJA, SA, GA 3 , ABA, ethylene, and mechanical damage) were significantly different, among which CYP89S1, CYP97B62 and squalene epoxidase(BpSE) and dammarenediol synthase $(B p D S)$ were highly expressed in leaves. $C Y P 89 S 1, C Y P 97 B 62$, and $C Y P 86 A 182$ genes are induced by MeJA and significant synergistic expression effects with lupeol synthase(BpW).CYP89S1, CYP97B62, CYP86A182 have C-28 oxidation function and catalyzing the conversion of lupeol to betulinic acid.Among them, CYP97B62 gene has the highest catalytic efficiency, increasing the content of betulinic acid by $1136 \%$.In addition, co expression of $B p M Y B 21$ and CYP86A182 can significantly enhance the conversion and synthesis efficiency of betulinic acid in tobacco(Nicotiana tabacum L.), and CYP89S1 can enhance salt and alkali resistance in yeast(Saccharomyces cerevisiae)
\end{abstract}

\section{Hosted file}

manuscript (20200407) . doc available at https://authorea.com/users/309679/articles/440563-threenew-cyp450-from-betula-platyphylla-suk-with-28-oxidation-function-catalyze-the-conversionof-lupineol-to-betulinic-acid 
(a)

CYP94B89[Betula platyphylla] CYP89S1[Betula platyphylla] CYP०A CYP716A52 2[Panax ginseng] CYP716A83[Centella asiatica] CYP716A154[Catharanthus roseus CYP716A140[Platycodon grandif CYP716A15[Vitis vinifera]

CYP716A180[Betula platyphylla] CYP716A12[Medicago truncatula CTP716A179[Glycyrrhiza uralens CYP716A78[Chenopodium quinoa] CYP716A110[Aquilegia coerulea] CYP716A2[Arabidopsis thaliana] CYP716A80[Barbarea vulgaris su CYP716Y1[Bupleurum CYPOAY12IVleurum califor CYP71D353[Lotus japonicus] CYP93E9[Phaseolus vulgaris] CYP93E4[Arachis hypogaea] CYP93E5[Cicer arietinum] CYP93E6[Glycyrrhiza glabra] CYP93E7[Lens culinaris] CYP93E8[Pisum sativum]

(b)

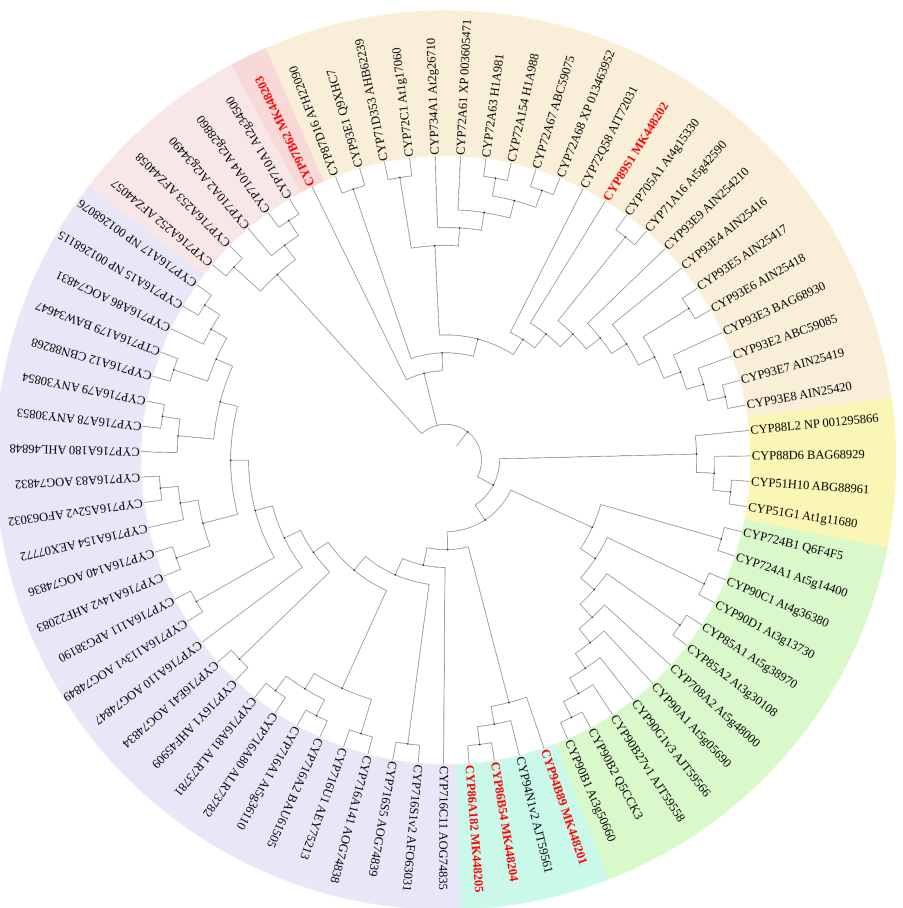



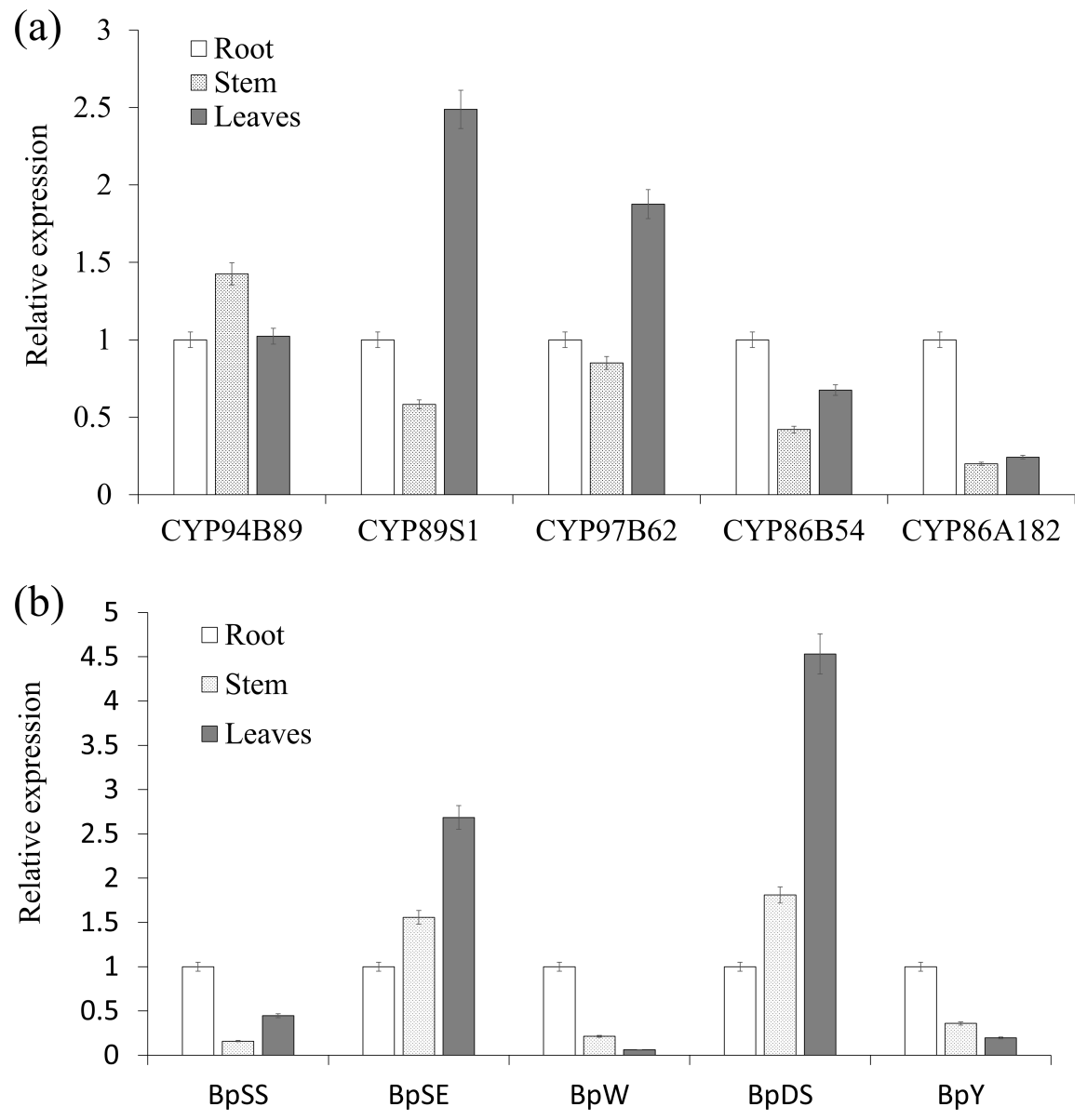


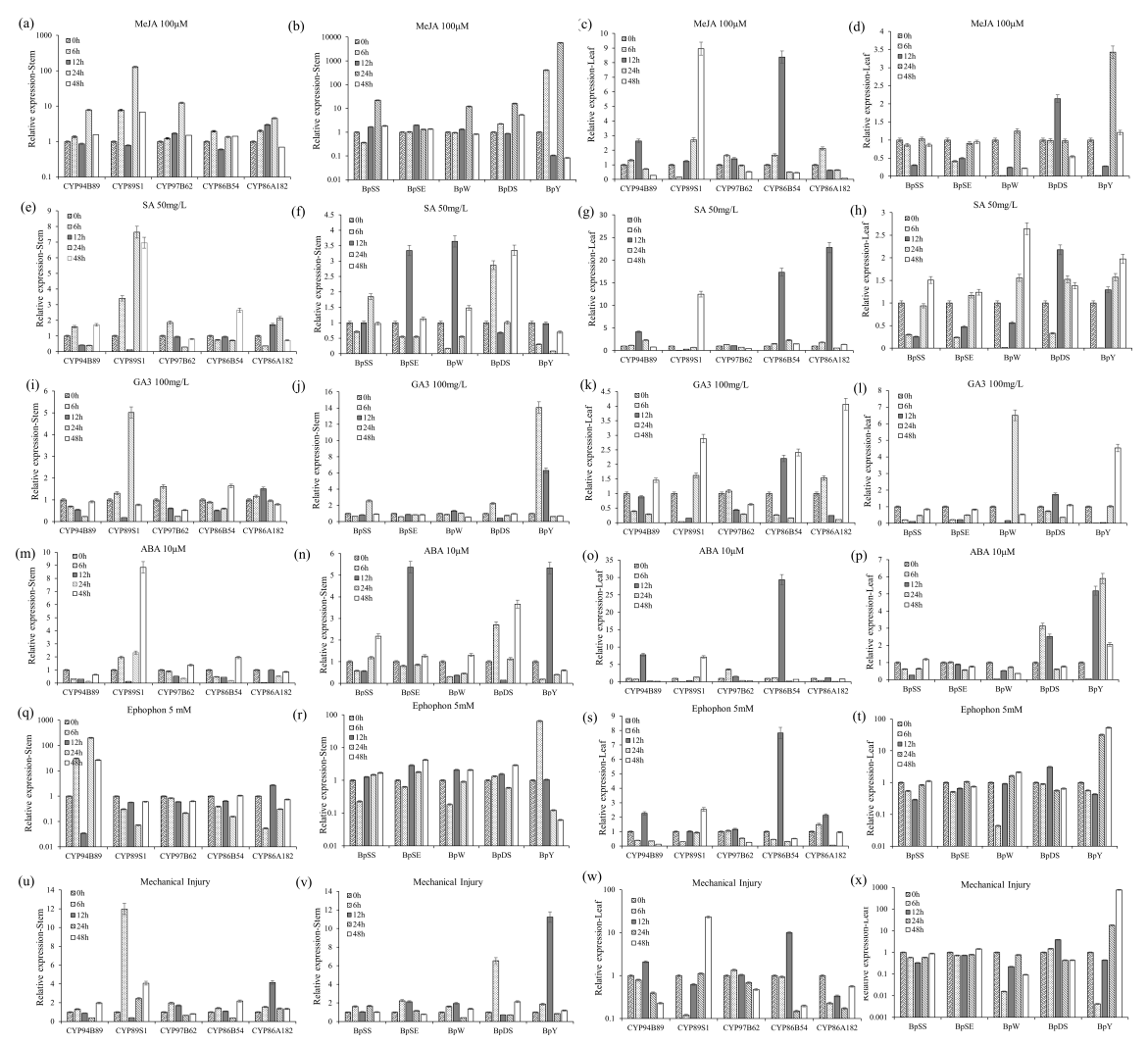



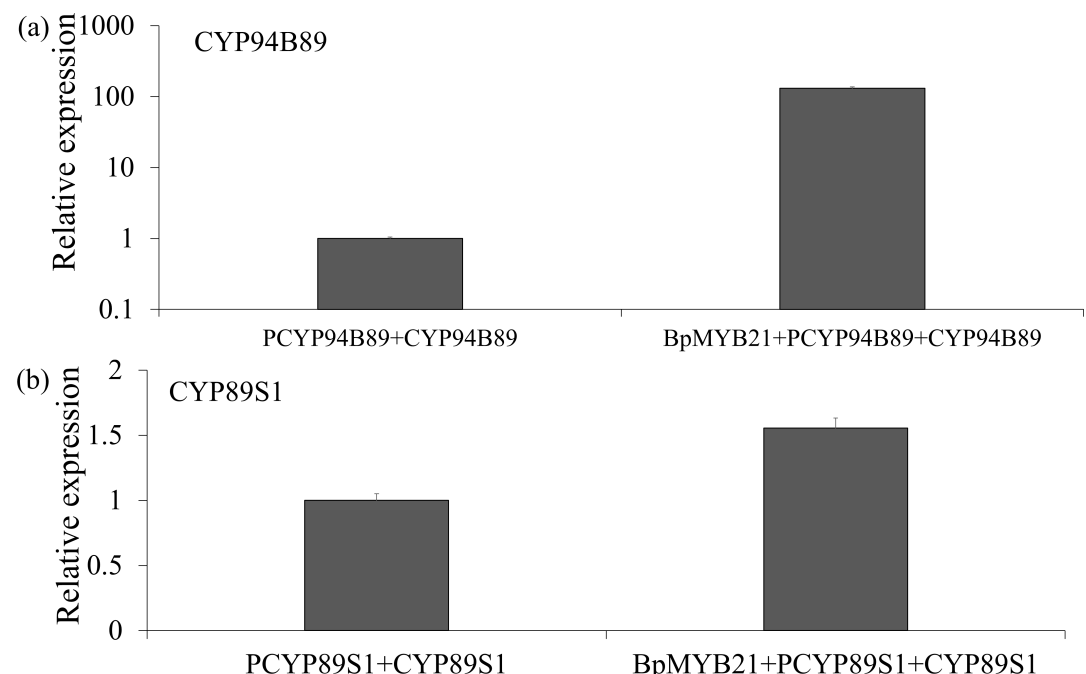

(c)
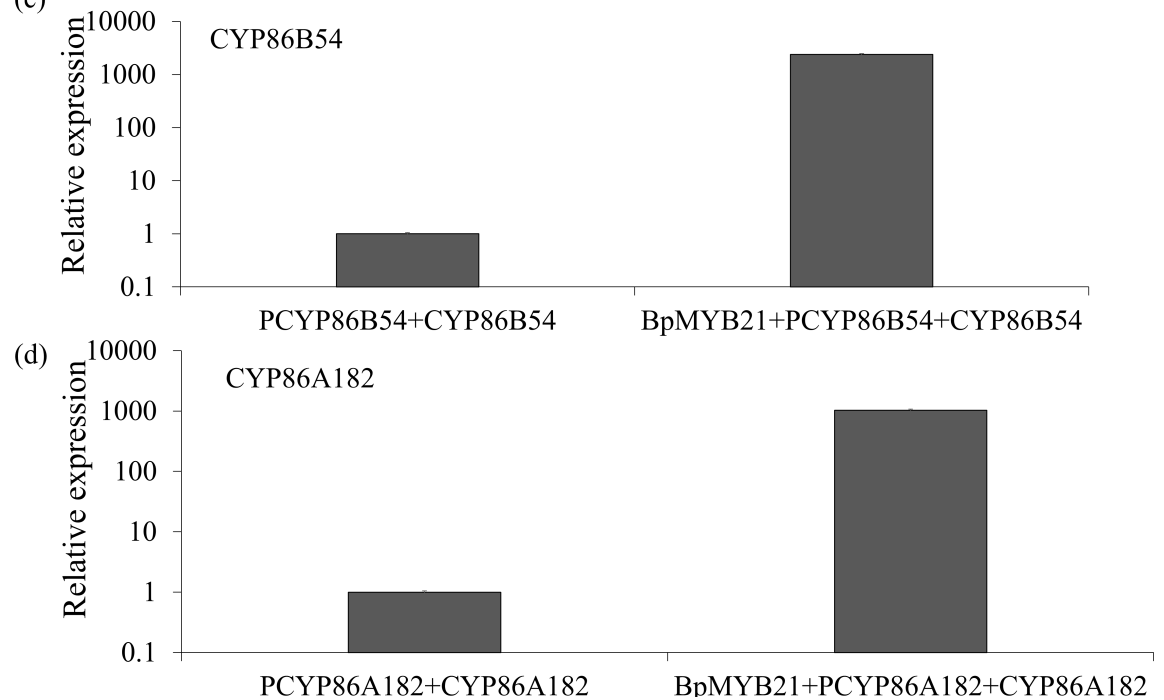

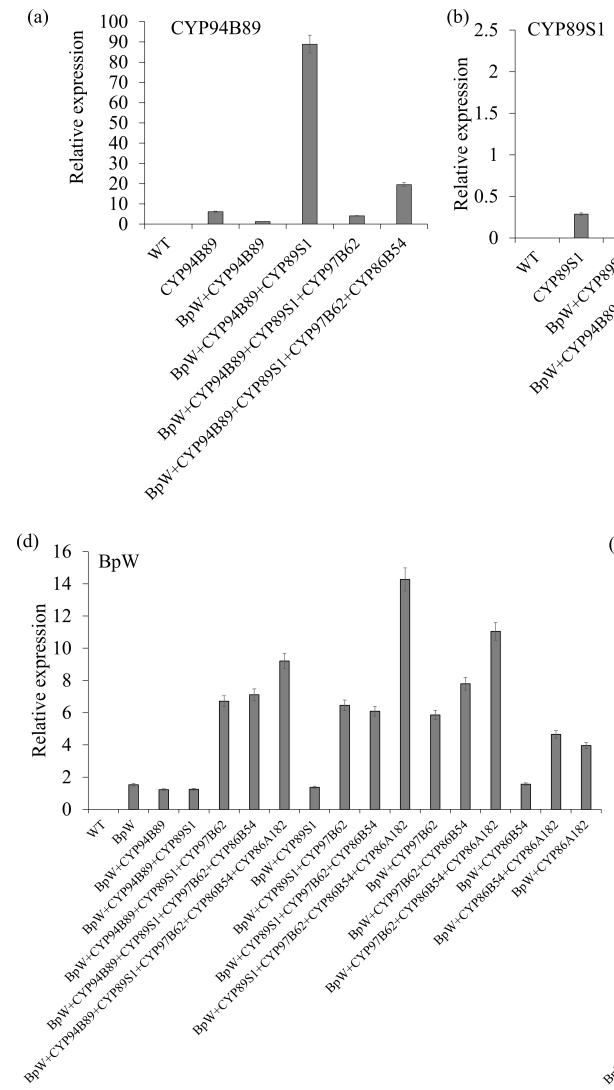
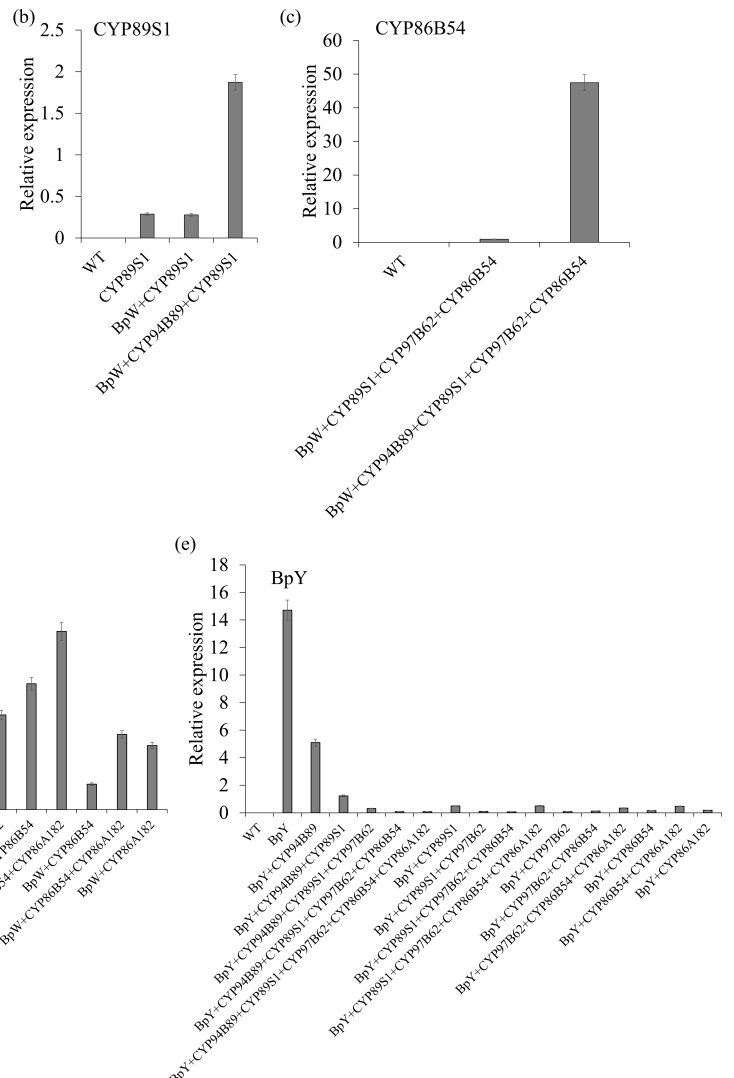

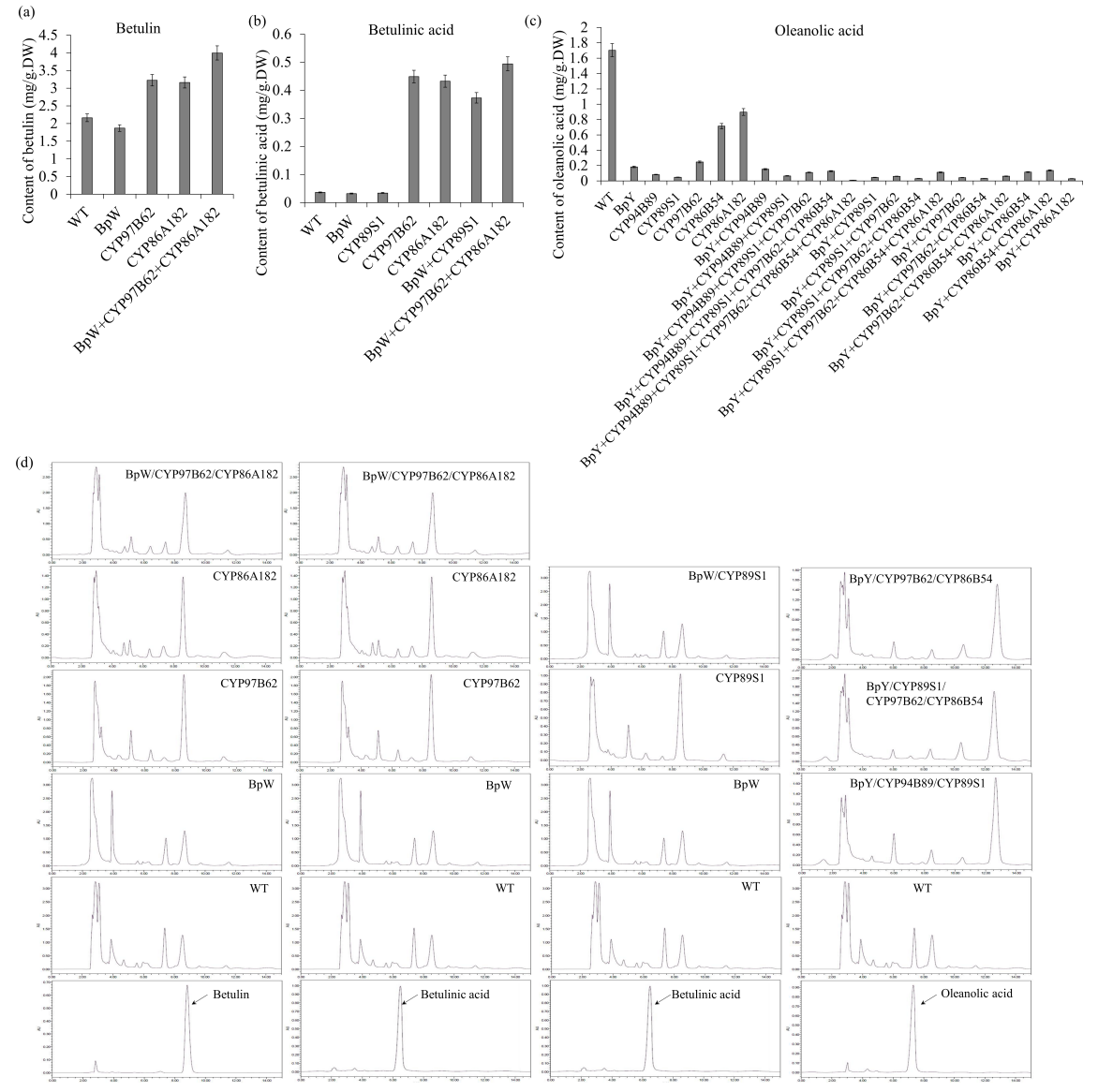

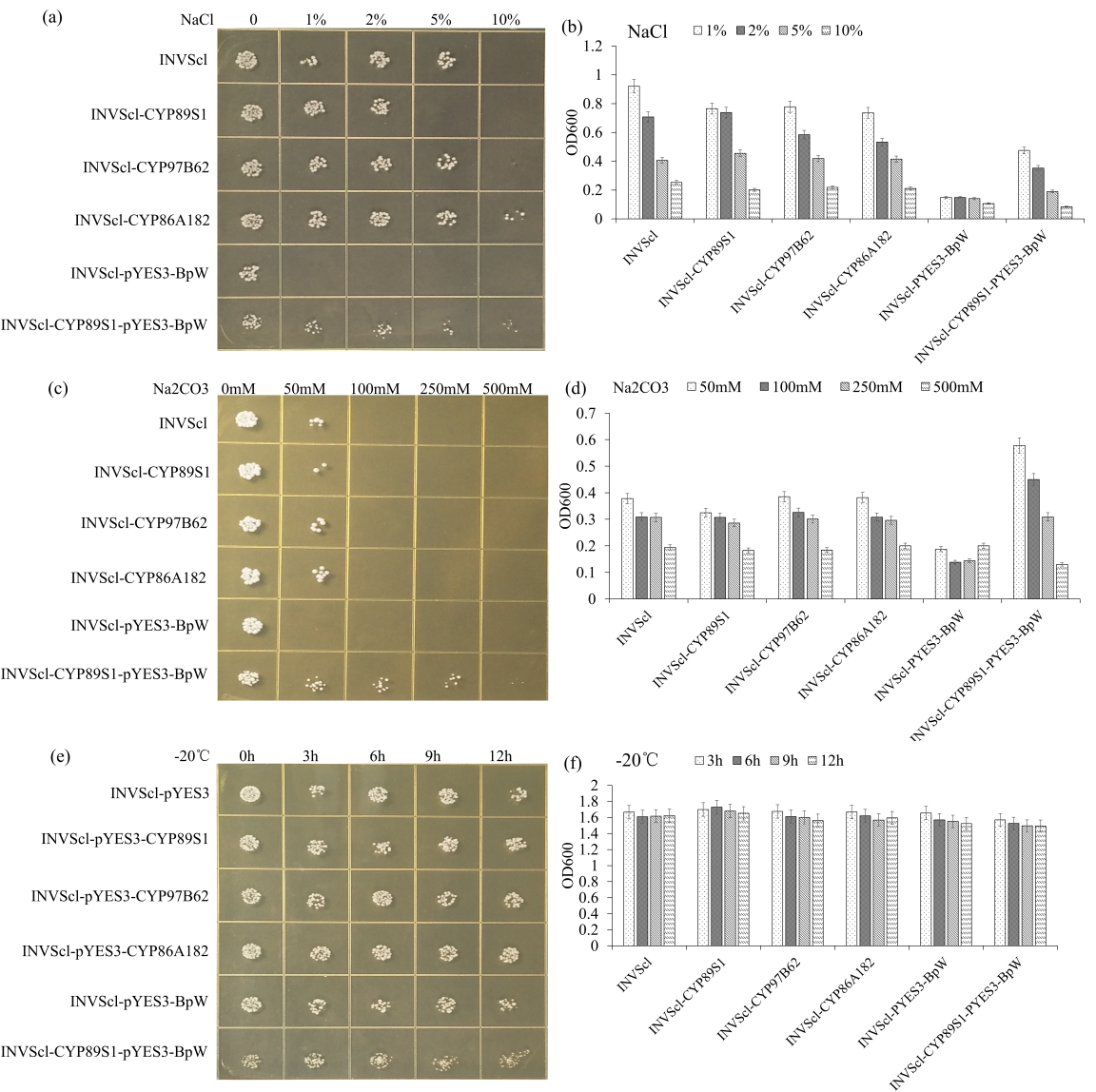


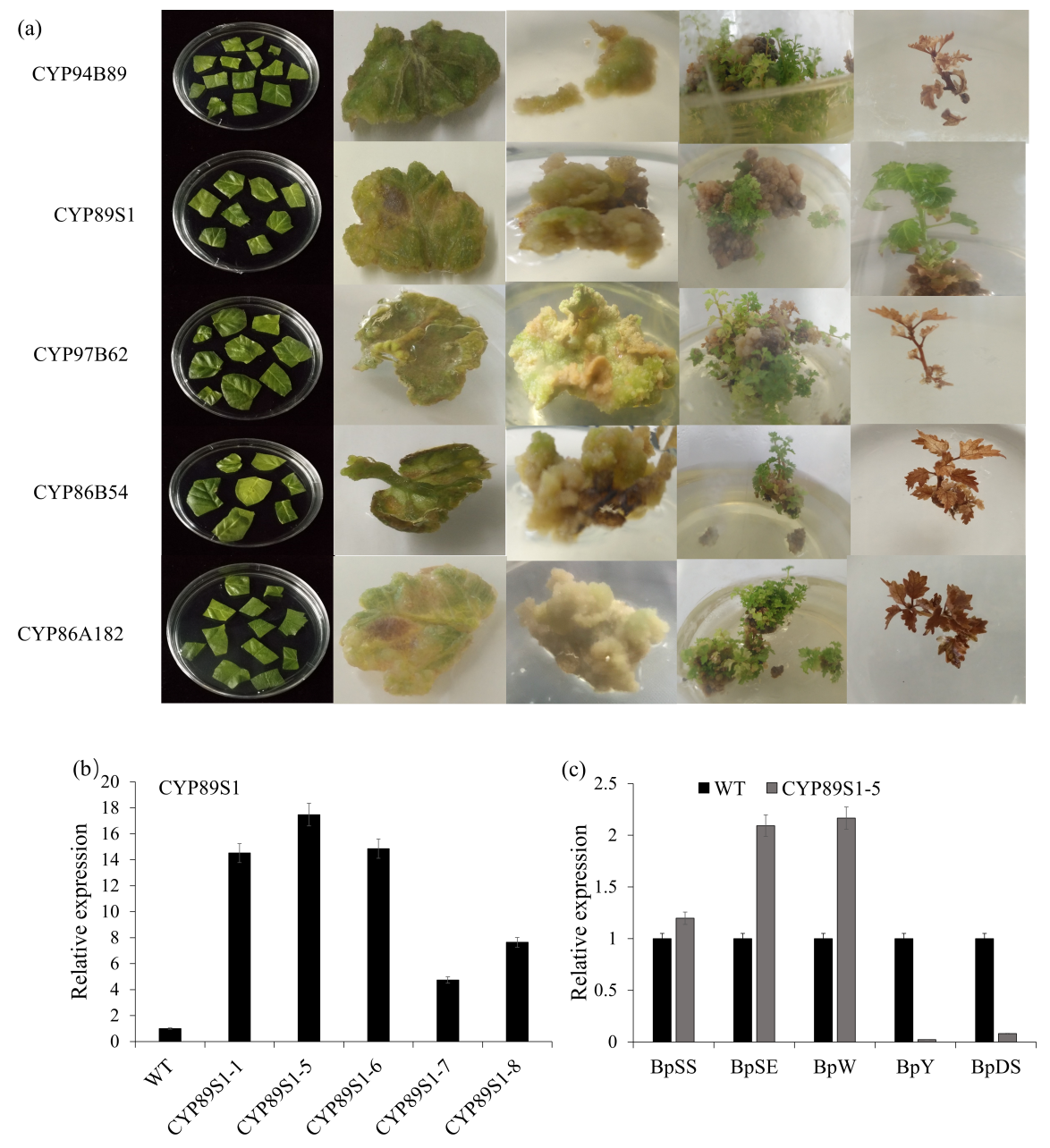




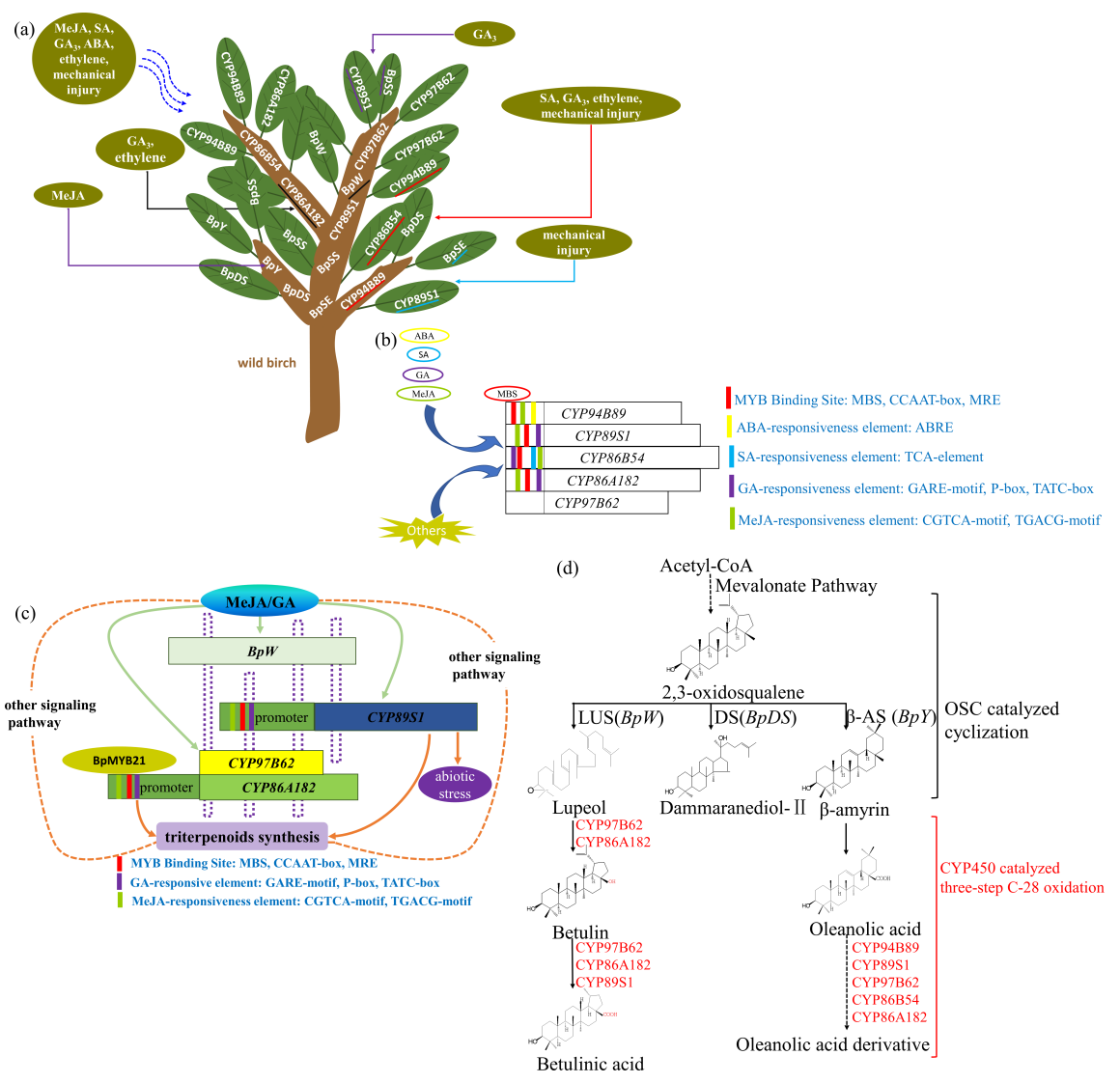

